\title{
Hernia de Amyand: un diagnóstico fortuito
}

\author{
Ana Paulina Pimienta-Sosa* \\ Cirugía, Hospital General de Occidente, Guadalajara, Jal., México
}

\section{ABSTRACT}

Amyand's hernia is an uncommon entity. It is an inguinal hernia that contains the cecal appendix, which căn be normal (usually), inflamed, and even perforated. Usually, the diagnosis is made during the surgical intervention. Amyand hernia is a rare condition that is estimated to account for approximately $1 \%$ of all inguinal hernias. Most of the cases reported present incarcerated or strangulated inguinal hernia characteristics. The diagnosis is $\frac{m a d e}{}$ during the transoperative period, when the surgical exploration is carried out. (Hosp Med Clin Manag. 2019;12:169-72) Corresponding author: Ana Paulina Pimienta-Sosa, anaps_8@ hotmail.com

Key words: Amyand hernia. Appendix. Surgery.

\section{RESUMEN}

La hernia de Amyand es una entidad infrecuente. Se trata de una hernia inguinal que contiene el apéndicescecal, que puede ser normal (lo más habitual) o estar inflamado, e incluso perforado. Habitualmente, el diagnóștico se realiza durante la intervención quirúrgica. La hernia de Amyand es una condición rara que se estima que reprèsenta aproximadamente el $1 \%$ de todas las hernias inguinales. La mayoría de los casos reportados presentan caräterísticas de hernia inguinal encarcelada o estrangulada. El diagnóstico es realizado durante el transoperatorio, al efectuarse la exploración quirúrgica.

Palabras clave: Hernia Amyand. Apéndice. Cirugía.

\section{INTRODUCCIÓN}

Un apéndice cecal dentro de una hernia inguinal, con o sin proceso inflamatorio, se denomina con la epónima Hernia de Amyand. El hallazgo de un apéndice cecal no inflamado dentro del saco peritoneal de una hernla inguinal indirecta fue descrito por primera vez por Garanğ en 1731'. Pero fue Claudio Amyand el primero en describir en 1735, una apendicetomía de la región inguinal de đün niño de 11 años de edad con fístula, debido a apễndicitis

\section{Correspondence:}

*Ana Paulina Pimienta-Sosa

E-mail: anaps_8@ hotmail.com
Date of reception: 04-07-2019

Date of acceptance: 28-08-2019

DOI: 10.24875/HMCM.19000217 
perforada en el saco herniario². Es una enfermedad de baja frecuencia, se estima que ocurre en el $1 \%$ de todas las hernias inguinales ${ }^{3}$.

En el caso de encontrar incidentalmente un apéndice normal dentro del saco herniario, realizar la apendicectomía profiláctica junto con la reparación de la hernia, no es conducta favorecida por muchos autores ${ }^{4,5}$. El estado inflamatorio del apéndice vermiforme determina el abordaje quirúrgico y el tipo de reparación de la hernia. Si existe apendicitis, la reparación de la hernia debe ser realizada con técnicas de Bassini o Shouldice, sin hacer uso de mallas o tapones sintéticos dentro del defecto debido al alto riesgo de infección de tales materiales protésicos ${ }^{6}$.

\section{HISTORIA}

Claudius Amyand (1660-1740), cirujano de origen francés e inglés por naturalización, que alcanzó el título de «sargento-cirujano» por el rey Jorge II de Inglaterra. Con su experiencia en cirugía de guerra, el 6 de diciembre de 1735 realizó la primera apendicectomía exitosa registrada. El paciente era un niño de 11 años con una fístula fecal entre el escroto y el muslo. La operación consistía en un apéndice crónicamente inflamado contenido dentro del saco de la hernia inguinal y perforada por un alfiler deglutido previamente. En la cirugía el apéndice fue extirpado, el muñón ligado y, debido a que gran parte del saco herniario se había separado de la piel y del conducto espermático, éste fue seccionado. El paciente se recuperó y se dio de alta ${ }^{7,8}$. Después de dos siglos, en 1937, motivado por la presentación de tres casos consecutivos de hernia de Amyand, el cirujano estadounidense William J. Ryan reunió y describió 11 casos de un universo de 8,962 de apendicitis; en todos los casos se encontró el apéndice en un saco herniario $(0.3 \%)^{9,10}$.

\section{EPIDEMIOLOGÍA}

La hernia de Amyand es una patología infrecuente ha sido reportada en pacientes que varían de 3 semanas a 92 años de edad ${ }^{5,11}$. El apéndice cecal no inflamado dentro de una hernia inguinal, se ha comunicado entre el $0.5 \%$ y el $1 \%$ de todas las hernioplastías en adultos, y el hallazgo de apendicitis aguda, en el $0.1 \%^{12}$. Es más frecuente en varones, con un promedio de edad de 42 años (aunque existen casos de pacientes pediátricos) ${ }^{8}$.

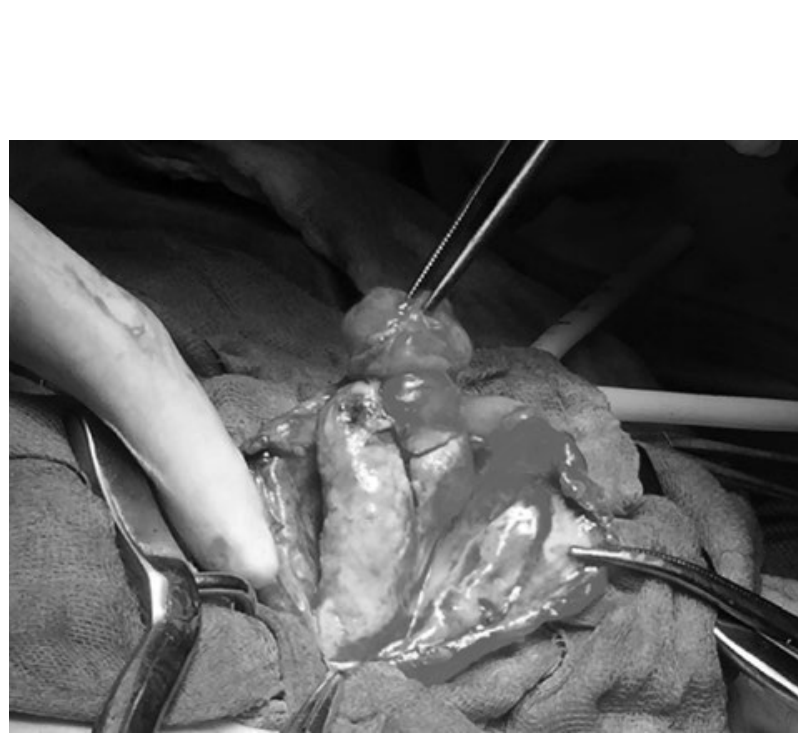

Figura 1. Hernia inguinal indirecta, dentro de saco herntiario. Se observa apéndice de aspecto gangrenoso.

\section{FISIOPATOLOGÍA}

La fisiopatología de la apendicitis aguda no estä clara. Muchos autores creen que existe una asociación entre el encarcelamiento e inflamación del apéndice ceeccal en el saco herniario, es decir, un fenómeno isquemico derivado de la compresión del órgano por el anillo ḥ̣erniario anterior al apéndice ${ }^{13}$. Se ha sugerido que êtapéndice se vuelva más vulnerable al trauma en la "Hernia de Amyand" y es finalmente retenido por adherehcias del saco herniario ${ }^{14}$. La contracción de los mûsculos abdominales y otros aumentos repentinos en la gresión intraabdominal pueden causar compresión del äapéndice, lo que resulta en la inflamación del mismo ${ }^{15}$ Posteriormente el suministro de sangre puede reducisse significativamente, resultando en la inflamación y el crecimiento excesivo de bacterias ${ }^{16}$.

\section{CLASIFICACIÓN}

La hernia de Amyand se subdivide en dos tiposinhernia inguinal directa e indirecta. Así mismo, el apéndice cecal puede encontrarse perfectamente normal o đencontrarse inflamado, con una complicación subsêcuente como perforación o absceso. También existe la laclasificación realizada por Losanoff (Tabla 1) que aş̧cia el tipo de hernia de Amyand con el tipo de tratamiento del apéndice cecal y la reparación herniaria realizada ${ }^{17,18}$.

La ausencia de cambios inflamatorios en el tipo $\stackrel{\bar{\varphi}}{\bar{\eta}}$, induce a la realización de hernioplastía electiva, utilizando una prótesis permanente. En tales casos lleva la expectativa 
Tabla 1. Clasificación de Losanoff para hernia de Amyand

\begin{tabular}{|c|c|c|c|c|c|c|}
\hline Tipo de hernia & 1 & 2 & 3 & 4 & $\stackrel{\complement}{\mathscr{\tau}}$ & \\
\hline Apéndice & Normal & $\begin{array}{c}\text { Apendicitis aguda sin } \\
\text { peritonitis }\end{array}$ & $\begin{array}{c}\text { Apendicitis aguda con } \\
\text { peritonitis }\end{array}$ & $\begin{array}{c}\text { Apendicitis agud } \\
\text { otra patología }\end{array}$ & a asöci & $\begin{array}{l}\text { lada a } \\
\text { inal }\end{array}$ \\
\hline Tratamiento & $\begin{array}{c}\text { Reducción o apendicectomía } \\
\text { (según la edad) } \\
\text { Hernioplastia con material } \\
\text { protésico }\end{array}$ & $\begin{array}{c}\text { Apendicectomía } \\
\text { Herniorrafía o hernioplastia } \\
\text { con material protésico }\end{array}$ & $\begin{array}{c}\text { Apendicectomía } \\
\text { Herniorrafía o hernioplastia } \\
\text { con material protésico }\end{array}$ & $\begin{array}{r}\text { Apendicec } \\
\text { Hernioplastia o } \\
\text { según se }\end{array}$ & 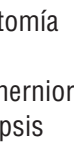 & \\
\hline
\end{tabular}

de incrementar la longevidad de la reparación porque evita la tensión sobre la sutura y elude los problemas metabólicos relacionados con la deficiencia de colágeno que se sabe que existe en pacientes con hernia ${ }^{19}$. La decisión de dejar o no un apéndice normal en este escenario clínico, se basa en criterios relacionados con la edad, esperanza de vida y el riesgo de desarrollar un cuadro agudo durante toda la vida. Sin embargo, la consideración de realizar apendicectomía en pacientes jóvenes debe ser contra el tamaño de la hernia, ya que el material protésico está relativamente contraindicado incluso en apendicectomía limpia electiva, mientras que las hernias de gran tamaño son más propensas a recurrir si se reparan usando solo tejido endógeno ${ }^{17}$.

Las hernias de Amyand tipo 2 son aquellas en las que los cambios sépticos se encuentran confinados al saco herniario y tienen una predisposición mayor a infección del material protésico ${ }^{20}$. Por lo tanto, las reparaciones con tensión o con colocación de prótesis de colágeno acelular (actualmente se piensa que son más resistentes a la infección que los materiales protésicos estándar) llevan la expectativa de una morbilidad menor, aunque a riesgo de una mayor recurrencia ${ }^{21,22}$.

La hernia tipo 3 representa un escenario donde la sepsis se ha extendido más allá del saco herniario y requiere cirugía más extensa. La conveniencia de realizar la reparación de la hernia en tales circunstancias depende de la magnitud de la sepsis y el estado del paciente; ambos reflejando la situación general y riesgo operativo. Es pertinente aplazar la hernioplastía si el paciente está gravemente enfermo o inestable ${ }^{23}$.

El tipo 4 de hernia de Amyand incluye todos los casos en los que una enfermedad grave y complicada coexiste fuera del saco herniario.

Con un alto índice de sospecha y contando con tomografía computarizada puede detectarse la coexistencia de tal patología, evitar retrasos, se puede detêctar la condición de coexistencia, evitar retrasos, ayuddar a planificar el procedimiento más adecuado, y así mejorar el resultado. Aquí también la hernioplastia puede estar contraindicada, si la condición del paciente es pobre o la esperanza de vida es limitada ${ }^{24}$.

\section{DIAGNÓSTICO}

El diagnóstico preoperatorio definitivo plantea uñesafío debido a signos y síntomas clínicos inespecíficộs. Los síntomas clínicos pueden ser engañosos y más ămmenudo se parecen a los de una hernia inguinal (estrangulada) con los signos y síntomas clásicos de apendicitis A veces una masa inguinal puede estar presente ${ }^{25}$. Eltyltrasonido es una excelente técnica para evaluar la región inguinal y se puede usar para detectar todos los tipos de hernias inguinales, incluida la hernia de Amyand Es seguro y barato, pero sigue siendo operador dependiente. La tomografía computarizada (TC) se puede utilizar para confirmar el diagnóstico, si es necesario. Al mismo tiempo, detectar posibles complicaciones intraabdominales como perforación y abscesos 26,27 .

\section{CONCLUSIÓN}

Para concluir, la hernia de Amyand es principalmente un hallazgo incidental durante la cirugía. El tratamiento depende de la elección personal del cirujano. Ed grado inflamatorio del apéndice determina el abordajeşquirúrgico y el tipo de reparación para el defecto herrniario. La exéresis de un apéndice sano está sujeto a clebate y no se ha alcanzado un consenso todavía.

\section{FINANCIAMIENTO}

El autor declara no recibir ningún financiamiento. 


\section{CONFLICTO DE INTERESES}

El autor declara no tener conflicto de intereses.

\section{BIBLIOGRAFÍA}

1. García-Cano E, Martínez-Gasperin J. Hernia de Amyand y apendicitis complicada; presentación de un caso y elección de tratamiento quirúrgico. Cir Ciruj. 2016;84(1):54-7.

2. Cigsar EB, Karadag CA, Docuku Al. Amyand's hernia: 11 years of experience. J Pediatr Surg. 2016;51(8):1327-1329.

3. Michalinos A, Vernadakis S. Amyand's hernia: a review. Am J Surg. 2014;207(6):989-95

4. Sharma H, Gupta A, Shekhawat NS, Memon B, Memon MA. Amyand's hernia: a report of 18 consecutive patients over a 15-year period. Hernia. 2007:11(1):31-5.

5. D'Alia C, Lo Schiavo MG, Tonante A, Taranto F, Gagliano E, Bonanno L, Di Giuseppe, G., Pagano, D., Sturniolo, G. Amyand's hernia: case report and review of the literature. Hernia. 2003;7(2):89-91.

6. Psarras K, Lalountas M, Baltatzis M, Pavlidis E, Tsitlakidis A, Symeonidis $\mathrm{N}$, et al. Amyand's hernia-a vermiform appendix presenting in an inguinal hernia: a case series. J Med Case Reports. 2011;5:463.

7. Amyand C. Of an inguinal rupture, with a pin in the appendix coeci, incrusted with stone, and some observations on wounds in the guts. Philos Transact R Soc Lon. 1736;39:329-36.

8. Milanchi S, Allins AD. Amyand's hernia: history, imaging, and management. Hernia. 2008:12:321-2.

9. Torres Hernández D, Roselló Fina JR, del Campo Abad R, Canals Rabasa PP, Enríquez Weinmann ES. Hernia de Amyand: presentación de un caso y revisión de la literatura. Arch Cir Gen Dig. 2003;22:1-5.

10. Ryan WJ. Hernia of the vermiform appendix. Ann Surg. 1937;106:135-9.

11. Anagnostopoulou S, Dimitroulis D, Troupis TG. Amyand's hernia: A case report. World J Gastroenterol. 2006:12:4761-3.
12. Kueper MA, Kirschniak A, Ladurner R, Granderath FA, Konigsrainer A. Incarcerated recurrent inguinal hernia with covered and perforated appendicitis and periappendicular abscess: Case report and review of the diterature. Hernia. 2007:11:189-91.

13. Weber RV, Hunt ZC, Kral JC. Amyand's hernia. Etiologic and therapeutic implications of two complications. Surg Rounds. 1999;22:552-6.

14. Abu-Dalu J, Urca I. Incarcerated inguinal hernia with a perforate@appendix and periappendicular abscess. Dis Colon Rectum. 1972;15:464-65.

15. Solecki R, Matyja A, Milanowski W. Amyand's hernia: a report of two cases. Hernia. 2003;7:50-1.

16. Singal R, Gupta S. "Amyand's Hernia" - Pathophysiology, role of investigations and treatment. Maedica (Buchar). 2011;6(4):321-7.

17. Losanoff JE, Basson MD. Amyand hernia: a classification to improve management. Hernia. 2008;12:325-6.

18. Agirre Etxabea L, Prieto Calvoa M. Hernia de Amyand (tipo 2 dedosanoff) diagnosticada preoperatoriamente y tratada mediante hernioplastia con malla biológica.

19. Bendavid R. The unified theory of hernia formation. Hernia. 2004:8:171176.

20. Livaditi E, Mavridis G, Christopoulos-Geroulanos G. Amyand's hernia in premature neonates: report of two cases. Hernia. 2007:11:547-549

21. Albo D, Awad SS, Berger DH, Bellows CF. Decellularized humancadaveric dermis provides a safe alternative for primary inguinal hernia repair in contaminated surgical fields. Am J Surg. 2006;192:e12-7.

22. Diaz JJ Jr, Guy J, Berkes MB, Guillamondegui 0, Miller RS. Acellutar dermal allograft for ventral hernia repair in the compromised surgicaFfield. Am Surg. 2006;72:1181-7.

23. Sharma H, Gupta A Shekhawat NS, Memon B, Memon MA Amyand's hernia: a report of 18 consecutive patients over a 15-year period. Hernia. 2007;11:31-5

24. Osorio JK, Guzman-Valdivia G. Ipsilateral Amyand's and Richter's hernia, complicated by necrosing fasciitis. Hernia. 2006:10:443-6.

25. Ivashchuk G, Cesmebasi A, Sorenson EP, Blaak C, Tubbs SR, Loukas M. Amyand's hernia: A review. Int Med J Exp Clin Res. 2014;20:140-6.

26. Mebis M, Hoste P, Jager T. Amyand's hernia. J Belg Soc Radiol. 2018; 102(1):8.

27. Sahu D, Swain S, Wani M, Reddy PK. Amyand's hernia: Our experience in the laparoscopic era. J Min Access Surg. 2015;11:151-3. 